\title{
Vertebral osteomyelitis caused by thoracic empyema, vice versa?
}

\author{
K.R. Liesker*, W.K. Taconis**, C.M.T. Plasmans+, A.J.M. Schreurs*
}

\begin{abstract}
Vertebral osteomyelitis caused by thoracic empyema, or vice versa? K.R. Liesker, W.K. Taconis, C.M.T. Plasmans, A.J.M. Schreurs. CERS Journals Ltd 1996.

ABSTRACT: Thoracic empyema and vertebral osteomyelitis is a rare combination. We report a case of vertebral osteomyelitis possibly caused by a progressive thoracic empyema.

The causative pathogen was Escherichia coli. Computed tomographic (CT) scan and magnetic resonance imaging (MRI) suggested the diagnosis of vertebral osteomyelitis, confirmed by transthoracic needle aspiration and operative findings. Aetiology and treatment are discussed.

Eur Respir J., 1996, 9, 2426-2428.
\end{abstract}

\begin{abstract}
Depts of *Pulmonology, **Radiology, +Orthopaedic Surgery, Onze Lieve Vrouwe Gasthuis, Amsterdam, The Netherlands.

Correspondence: A.J.M. Schreurs, Dept of Pulmonology, P.O. Box 95500, 1090 HM Amsterdam, The Netherlands
\end{abstract}

Keywords: Escherichia coli, thoracic empyema, vertebral osteomyelitis

Received: October 151995

Accepted after revision February 131996
Thoracic empyema is a rare complication of vertebral osteomyelitis, but has been reported before [1-3]. Both conditions can occur as a result of haematogenous seeding of microorganisms from a different source. Depending on the primary site, different pathogens are responsible for the infection [4]. In this case report, the close proximity of the pleural empyema and the infected vertebral bodies suggests a direct relationship. We assume that our patient had vertebral osteomyelitis caused by a progressive thoracic empyema.

\section{Case report}

A 63 year old woman presented with dyspnoea, fever and right-sided chest pain. Previously, she had been treated by her general practitioner with amoxycillin for cystitis. There was no cough, no sputum production and no haemoptysis. Her medical history revealed recurrent cystitis and non-insulin-dependent diabetes mellitus.

Physical examination showed an obese female: rectal temperature was $38.6^{\circ} \mathrm{C}$; blood pressure $140 / 80 \mathrm{mmHg}$; a regular pulse of 96 beats $\mathrm{min}^{-1}$. Dullness of percussion and diminished breath sounds were noted over the lower part of the right hemithorax. Examination of the spine revealed no abnormalities. Laboratory studies disclosed: a haemoglobin level of $6.9 \mathrm{mmol} \cdot \mathrm{L}^{-1}$; white blood cell (WBC) count $11 \times 10^{9}$ cells $\cdot \mathrm{L}^{-1}$ with a normal differential count; erythrocyte sedimentation rate (ESR) $122 \mathrm{~mm} \cdot \mathrm{h}^{-1}$; alkaline phosphatase $105 \mathrm{U} \cdot \mathrm{L}^{-1}$; and $\gamma$-glutamyl transferase $(\gamma-\mathrm{GT}) 122 \mathrm{U} \cdot \mathrm{L}^{-1}$. A chest radiograph revealed opacification of the right middle and lower lung fields and a considerable amount of pleural fluid.

Thoracocentesis produced clear yellow fluid with: protein $53 \mathrm{~g} \cdot \mathrm{L}^{-1}$; lactate dehydrogenase $(\mathrm{LDH}) 900 \mathrm{U} \cdot \mathrm{L}^{-1}$; glucose $11.3 \mathrm{mmol} \cdot \mathrm{L}^{-1}$; and amylase $22 \mathrm{mmol} \cdot \mathrm{L}^{-1}$. Gram stain revealed many neutrophils, but no bacteria were isolated. Ziehl-Neelsen staining was negative and cytological examination showed no malignant cells. A purified protein derivative (PPD) (tuberculin) skin test was negative.
Six days after hospitalization, a computed-tomography (CT) scan of the thorax (fig. 1) established loculated pleural fluid, pleural thickening, and partial collapse of the right lower lobe. The thoracic vertebrae were intact. With the aid of this CT scan and thoracic ultrasound, thoracocentesis was repeated several times and eventually revealed pus. Culture showed an amoxycillin-sensitive Escherichia coli. Treatment with amoxycillin was started intrapleurally and intravenously. The temperature subsided and the patient recovered gradually in the next 3 weeks, after which the antibiotics were continued orally.

After discharge from hospital, recurrent progressive dyspnoea, fever and intense medial and right-sided thoracic pain were the reasons for a new consultation, nearly 3 months after the first presentation. Physical examination now revealed a tenderness to percussion over the thoracic spine. Neurological examination was normal.

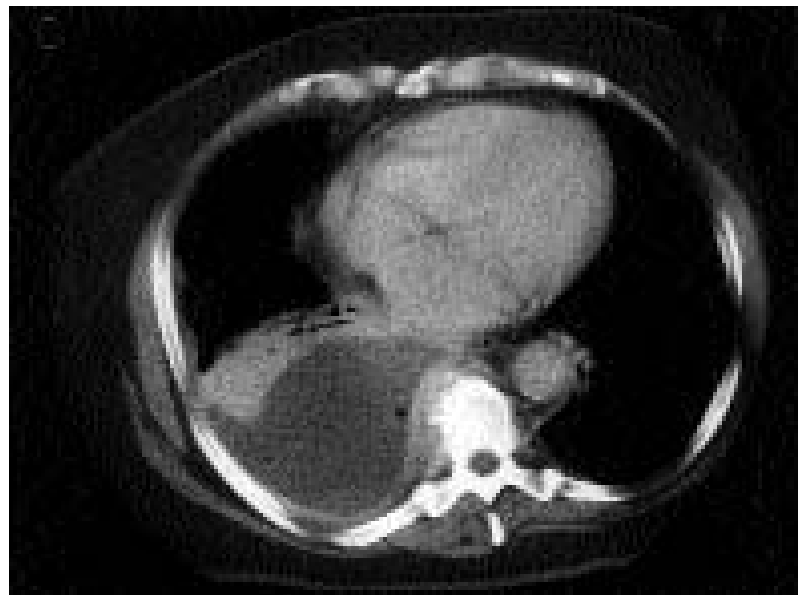

Fig. 1. - Computed tomographic (CT) image of the thorax (with intravenous contrast), at first presentation. The right hemithorax demonstrates loculated pleural fluid with thickening of the pleura. There is a small air bubble which seems to be trapped. Both findings are compatible with empyema. Furthermore, there is atelectasis of the right lower lobe. The thoracic vertebra on this level appears to be intact. 
A new CT scan of the thorax (fig. 2a) showed, in addition to right- and left-sided pleural fluid, a paravertebral soft tissue swelling with destruction of thoracic vertebrae. A CT scan and magnetic resonance imaging (MRI) of the spine (fig. 2b) confirmed a spondylodiscitis of the thoracic vertebrae 9 and 10 .

a)

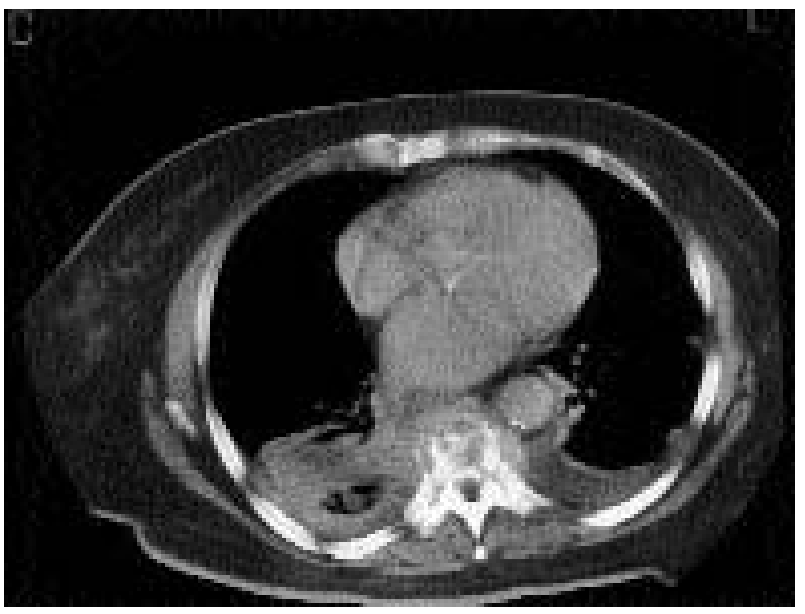

b)

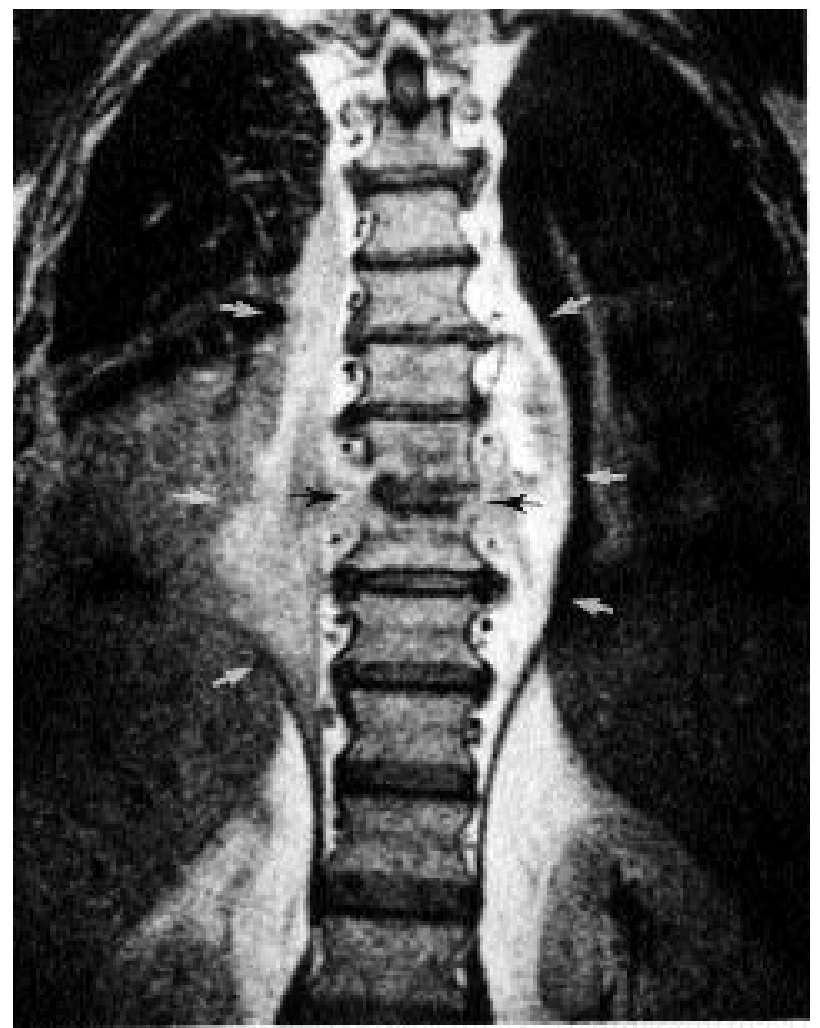

Fig. 2. - a) Computed tomographic (CT) scan made 3 months after the first presentation, at the same level as figure 1 . The right hemithorax shows some air, possibly due to puncture. There is also fluid in the left hemithorax. There is now obvious destruction of the vertebral body of Th. 9 and a prevertebral soft tissue swelling. b) Magnetic resonance imaging (MRI) made on the same day as figure 2a. Coronal T1 (TR 550, TE 22) weighted image (after intravenously injected gadolinium diethylenetriamine penta-acetate (DTPA)) shows destruction of the intervertebral space between thoracic vertebrae 9 and 10 (Th.9-10) (black arrows) and of the adjacent vertebrae. There is a huge soft tissue swelling (white arrows), extending proximal to the level of Th. 6 and distal to Th. 11 .
Thoracocentesis produced pus. Escherichia coli was again isolated, but was now resistant to amoxycillin. The same bacteria were isolated from urine and blood cultures. Examination of the left-sided pleural fluid revealed no pathogen. The patient was treated with cefotaxime intravenously and underwent surgery a few days later. Although there was considerable loss of bone of the vertebral bodies, there were no signs of a compression fracture or of vertebral body collapse with angulation. There were no signs of spinal cord compression and a decompressive surgical procedure was not needed. Because of the extensive destruction and soft tissue extension, debridement with bone grafting was carried out. Fibrinopurulent material and pus were evacuated via a right posterolateral thoracotomy. The affected vertebral bodies were debrided and iliac crest grafts and cancellous bone were inserted. A tube was inserted in the left hemithorax for drainage. Histological examination of the removed vertebral bodies showed necrotizing infection. The same Escherichia coli strain was isolated from all affected material.

Postoperatively, the patient was treated with intravenous antibiotics. Two months after the first operation, a posterior spondylodesis was performed to stabilize the affected spine. The patient had an uneventful recovery, the temperature remained normal and the ESR dropped to $12 \mathrm{~mm} \cdot \mathrm{h}^{-1}$ on the day of discharge, nearly 7 months after first presentation.

\section{Discussion}

Since we isolated the same bacteria in urine, blood, affected vertebrae and pleural fluid, it was clear that the primary source of infection was urinogenital with haematogenous spread during bacteraemia.

The most interesting question, however, is whether the vertebral osteomyelitis was a complication of the thoracic empyema or vice versa. In other words, direct spread from the pleura to the adjacent vertebrae or from the vertebrae to the pleura. A third possibility is haematogenous spread from the urinary tract both to pleura and vertebrae.

Although, theoretically, haematogenous spread seems the most likely cause and direct spread from the vertebrae to the pleura has been reported previously [1-3], we suggest that the primary source of infection was the thoracic empyema causing the vertebral osteomyelitis per continuitatum. This opinion is supported by the clinical picture and the sequence of events. Firstly, our patient started to have back pains only 3 months after her first hospitalization. Secondly, CT scans of the thorax made 1,3 and 6 weeks after first presentation showed no sign of vertebral osteomyelitis. However, the last CT scan revealed a progressive pleural infection expanding towards the spine as a prevertebral mass, and signs of pleural effusion on the left side, which were not present at previous CT scans. Thirdly, during the conservative treatment, the ESR did not normalize and CT scans showed only partial resolution of the pleura-pulmonary abnormalities, indicating a slow response to therapy. Although the radiographically demonstrable changes of vertebral osteomyelitis may lag behind the clinical findings by weeks or months [2, 4-9], it seems unlikely that a progressive vertebral osteomyelitis causing thoracic empyema, would not be seen on a CT scan made 6 weeks after the first 
signs of the empyema. However, it is important to note that initially only routine CT scans of the thorax were made, with one centimetre slice thickness, and no skeletal windows or level settings were used.

Vertebral osteomyelitis is usually caused by the haematogenous spread of microorganisms to the richly vascularized vertebral end-plate, with which urinary tract infections are traditionally associated [4]. Rarely, it can occur by direct contamination, as in this case. Escherichia coli is occasionally the pathogen causing empyema, most frequently as a result of aspiration pneumonia, oesophageal pathology or subdiaphragmatic abscess. Other frequent aetiological pathogens are Staphylococcus aureus and Streptococcus pneumoniae [10-12]. The most common predisposing conditions of thoracic empyema are primary bronchopulmonary infections, thoracic surgery, and oesophageal or subdiaphragmatic abscess [1, 10-12].

In this case, the primary source of infection was probably a recurrent cystitis, in a predisposed diabetic patient. Bacteriaemia caused a thoracic empyema, which in its turn led to vertebral osteomyelitis. Therapy was instituted and the patient fully recovered after 9 months.

\section{References}

1. Thurer RJ, Palatianos GM. Surgical aspects of the pleural space. Semin Respir Med 1987; 9: 98-115.
2. Göb A, Müller RT. Unspezifische Spondylitis. In: Witt AN, Rettig H, Schlegel KF, eds. Orthopädie in Praxis und Klinik. 2nd edn. Stuttgart, Georg Thieme Verlag, 1990; 9: pp. 120-139.

3. Hoeper MM, Blauth MB, Schaefers HJ. Increasing back pain in a young man. Chest 1993; 104: 925-926.

4. Waldvogel FA, Vasey H. Osteomyelitis: the past decade. N Engl J Med 1980; 303: 360-370.

5. Wineski RJ. Infectious disease of the spine. Orthop Clin North Am 1991; 22: 491-501.

6. Musher DM, Thorsteinsson SB, Minuth SN, Luchi RS. Vertebral osteomyelitis: still a diagnostic pitfall. Arch Intern Med 1976; 136: 105-110.

7. Scully RE, Mark EJ, McNeely WF, McNeely BU. Case records of the Massachusetts General Hospital (Case 241989). N Engl J Med 1989; 320: 1610-1618.

8. Sapico FL, Montgomerie JZ. Vertebral osteomyelitis in intravenous drug abusers: report of three cases and review of the literature. Rev Infect Dis 1980; 2: 196-206.

9. Burke DR, Brant-Zawadzki M. CT of pyogenic spine infection. Neuroradiology 1985; 27: 131-137.

10. Brook I, Frazier EH. Aerobic and anaerobic microbiology of empyema. Chest 1993; 103: 1502-1507.

11. Lemmer JH, Botham MJ, Orringer MB. Modern management of adult empyema. J Thorac Cardiovasc Surg 1985; 90: 849-855.

12. Varkey B, Rose HD, Kutty CPK, Politis J. Empyema thoracis during a ten year period. Arch Intern Med 1981; 141: 1771-1776. 